\title{
Antibody-CD20-interferon-alpha fusion protein has superior in vivo activity against human $B$ cell lymphomas compared to Rituximab, and enhanced complement-dependent cytotoxicity in vitro
}

\author{
Reiko Yamada', Kristopher Steward ${ }^{1}$, Gataree Ngarmchamnanrith ${ }^{1}$, Ryan Trinh ${ }^{2}$, Sanjay Khare ${ }^{3}$, Raj Sachdev ${ }^{3}$, \\ Iqbal Grewal ${ }^{3}$, Sherie Morrison², John Timmerman ${ }^{1 *}$
}

From Society for Immunotherapy of Cancer 28th Annual Meeting

National Harbor, MD, USA. 8-10 November 2013

\section{Background}

We previously reported an anti-CD20-interferon-alpha $(\mathrm{IFN} \alpha)$ fusion protein able to induce apoptosis and promote in vivo eradication of a human CD20expressing mouse B cell lymphoma [Xuan et al, Blood 2010]. We now report the activity of a recombinant anti-CD20-human IFN $\alpha$ fusion protein against human non-Hodgkin B cell lymphomas (NHL).

\section{Methods}

Anti-CD20-hIFN $\alpha$ was evaluated against a panel of human Burkitt, diffuse large B cell (DLBCL), and mantle cell lymphoma cell lines. Proliferation was measured by [3H]-thymidine, complement-dependent cytotoxicity (CDC) by PI flow cytometry, and antibody-dependent cellular cytotoxicity (ADCC) by LDH release using PBMC effectors. NHL xenografts were grown in SCID mice.

\section{Results}

Anti-CD20-hIFN $\alpha$ induced stronger growth inhibition than rituximab, particularly against Burkitt and germinal center-type DLBCL NHLs. Tumor growth inhibition by anti-CD20-hIFN $\alpha$ was associated with substantial apoptosis in some cell lines. Anti-CD20-hIFNa exhibited potent ADCC activity against Daudi, Ramos, and Raji cells, identical to rituximab. Surprisingly, anti-CD20-hIFN $\alpha$ exhibited superior CDC compared to rituximab against Daudi, Ramos, and Raji cells that was dependent upon linkage of IFN $\alpha$ to the anti-CD20 antibody, and correlated with improved complement fixation. Importantly, against Raji NHL xenograft tumors in SCID mice, anti-CD20-hIFNa achieved superior efficacy compared to rituximab $(\mathrm{p}=0.0015)$ and control fusion protein $(\mathrm{p}<0.0001)$. At antibody doses at which Raji xenograft tumors progressed through rituximab, anti-CD20-hIFN $\alpha$ eradicated 50-88\% of established tumors. Non-targeted control fusion protein had only minor effects on tumor growth.

\section{Conclusions}

Anti-CD20-hIFN $\alpha$ has stronger direct anti-proliferative and CDC activities than rituximab against human NHL while retaining potent ADCC activity, and also has the ability to eradicate established NHL xenografts in vivo. These results support the further development of antiCD20-hIFN $\alpha$ for the treatment of B cell NHL, and a phase I first-in-human clinical trial is currently being planned.

\section{Authors' details}

${ }^{1}$ Medicine - Hematology/Oncology, UCLA, Los Angeles, CA, USA.

${ }^{2}$ Microbiology, Immunology, and Molecular Genetics, UCLA, Los Angeles, CA, USA. ${ }^{3}$ ImmunGene, Inc. and Valor Biotherapeutics, Thousand Oaks, CA, USA.

Published: 7 November 2013

doi:10.1186/2051-1426-1-S1-P263

Cite this article as: Yamada et al:: Antibody-CD20-interferon-alpha fusion protein has superior in vivo activity against human B cell lymphomas compared to Rituximab, and enhanced complementdependent cytotoxicity in vitro. Journal for ImmunoTherapy of Cancer 2013 1(Suppl 1):P263.

'Medicine - Hematology/Oncology, UCLA, Los Angeles, CA, USA

Full list of author information is available at the end of the article

C 2013 Yamada et al; licensee BioMed Central Ltd. This is an Open Access article distributed under the terms of the Creative Commons 